\author{
Adela Parra Romero \\ Universidad Icesi, Colombia \\ adela.parra@correo.icesi.edu.co \\ Zeneire Cadena Díaz \\ Universidad Icesi, Colombia \\ zejac@yahoo.com
}

\title{
El medio ambiente desde las relaciones de ciencia, tecnología y sociedad: un panorama general ${ }^{1}$
}
The environment and the relations of science, technology and society: a general overview

O meio ambiente a partir das relaçôes da ciência, tecnologia e
sociedade: um panorama geral

Artículo de investigación científica recibido el 28/07/2010 y aprobado el 02/11/2010

1 Este artículo hace parte del proyecto de investigación: "Programa CTS: balance y estado documental alrededor de temas estratégicos -redes e innovación, medio ambiente; economía y desarrollo; cultura, comunicación y educación”, realizado por el grupo en Ciencia, Tecnología y Sociedad, de la Facultad de Derecho y Ciencias Sociales de la Universidad Icesi, durante el año académico 2009. 



\title{
Resumen
}

En el tema del medio ambiente, la ciencia y la tecnología han jugado un doble papel en la forma de abordarlo. En primer lugar, se ha culpado a la ciencia y a la tecnología de provocar la mayor parte de los problemas que actualmente aquejan al planeta. En segundo lugar, se le otorga la responsabilidad de crear soluciones para estos problemas, a partir de la creación de nuevas tecnologías, del cambio en los patrones de consumo y de la exploración de alternativas para la satisfacción de las necesidades de las personas. Este artículo hace una revisión desde la perspectiva CTS (ciencia, tecnología y sociedad) de los principales temas en el estudio de la tecnología y el medio ambiente.

Palabras clave: Relaciones CTS, Medio ambiente

\begin{abstract}
Science and technology have played a dual role in the way they approach the topic of the environment. First of all, science and technology have been blamed of causing most of the problems currently faced by our planet. Secondly, they are also given the responsibility of creating solutions for these problems, by developing new technologies, changing consumption patterns and exploring alternatives to meet people needs. This article reviews the main topics in the study of technology and environment from the perspective of STS (science, technology and society).
\end{abstract}

Key words: STS Relationships, Environment

\section{Resumo}

No tema do meio ambiente, a ciência e a tecnologia tiveram um duplo papel na forma de o abordar. Em primeiro lugar, se culpou a ciência e a tecnologia de provocarem a maior parte dos problemas que atualmente agridem o planeta. Em segundo lugar, é-lhe dada a responsabilidade de criar soluçóes para estes problemas a partir da criação de novas tecnologias, de mudança nos padróes de consumo e da exploraçáo de alternativas para a satisfação das necessidades das pessoas. Este artigo faz uma revisão a partir da perspectiva de CTS (ciência, tecnologia e sociedade) dos principais temas no estudo da tecnologia e o meio ambiente.

Palavras-chave: Relaçóes de CTS, Meio ambiente 



\section{Introducción}

El movimiento CTS se orienta al estudio, desde la interdisciplinariedad, de las relaciones entre la ciencia, la tecnología y la sociedad. El estudio de estas relaciones, que se presentan de manera compleja y no lineal, da origen a dos perspectivas: la primera se orienta hacia las implicaciones de la ciencia y la tecnología sobre los sistemas sociales y, la segunda, a cómo la sociedad y sus problemas pueden influenciar la construcción del conocimiento científico y tecnológico. Específicamente, las repercusiones de la tecnología sobre el medio ambiente ha sido uno de los temas principales del movimiento CTS bajo la primera perspectiva.

Ante la ocurrencia de desastres relacionados con el desarrollo industrial como derrames de petróleo o catástrofes nucleares, se comienza a formar una concienciación colectiva acerca de los riesgos e impactos que podrían generar una ciencia y tecnología fuera de control. Igualmente, fenómenos como el cambio climático, la contaminación de las fuentes de agua y la pérdida de biodiversidad, entre otros, han llamado la atención sobre la necesidad de desarrollar tecnologías que produzcan mínimos dańos al medio ambiente. A la vez que se privilegie el uso de estas tecnologías frente aquellas que generan un alto impacto negativo al ambiente.

Como consecuencia, la ciencia y la tecnología se transforman en objeto de análisis y debate político. Y las políticas científico-tecnológicas están siendo profundamente revisadas por los gobiernos. Una revisión que hace énfasis en el control y la participación pública en el desarrollo científico-tecnológico. Sin embargo, la pregunta que subyace a este planteamiento es: ¿sobre quién recae la responsabilidad de orientar la ciencia y la tecnología?, ya que en la mayoría de los casos los intereses de los investigadores, por un lado, y de las sociedades, por el otro, no necesariamente coinciden (Antal, 2007). ${ }^{2}$

Al hablar del control en el desarrollo, también cabe preguntarse: ¿cuáles son los criterios y cuál es su fuerza o contundencia, para elegir determinada tecnología? ¿Serían determinantes solamente los criterios ambientales y técnicos? Y, una vez seleccionada una tecnología, ¿qué pasa con los impactos generados? ¿A quién se atribuyen los beneficios y los riesgos asociados?

Con estas cuestiones de fondo, el propósito de este trabajo es presentar algunas de las herramientas conceptuales, que desde el análisis de las relaciones de la ciencia, la tecnología y la sociedad, se disponen para su tratamiento. El resultado se puede catalogar como un panorama general, que le permita a un lector

2 En la actualidad los estudios sociales de la ciencia y la tecnología indagan por las posibles características que deberían tener las sociedades para asumir la responsabilidad de participar en la toma de decisiones en cuestiones tecno-científicas. De igual manera, realizan cuestionamientos acerca de las capacidades de las mismas para cumplir estas responsabilidades 
interesado encontrar los lineamientos y los autores destacados en cada temática. Debe advertirse que, debido a la amplitud y complejidad del tema, este artículo no se detiene explícitamente en el contexto regional, nacional ni mucho menos latinoamericano. Este aspecto se espera abordar en una próxima indagación.

En la primera parte del artículo se describen las principales preocupaciones acerca del medio ambiente y su relación con el progreso científico y tecnológico. En la segunda parte, siendo el principal interés del trabajo, se presentan las tendencias y las categorías conceptuales desde la perspectiva CTS para resolver las preocupaciones descritas en la primera parte. Los temas tratados son: la gestión del riesgo, la evaluación constructiva de tecnologías (ECT), la participación y la percepción pública y, finalmente, la educación ambiental.

Cabe resaltar que la revisión realizada para este trabajo toma en consideración los principales expositores de los temas. Al igual que los planteamientos y cuestionamientos fundamentales a cada concepto. Así el lector podrá encontrar en este artículo una revisión no exhaustiva sin enfatizar en los contextos latinoamericanos y, por ahora, sin ahondar en las cuestiones que presuponen la aplicación de estos temas precisamente a nivel regional.

Con esta aclaración de base, se muestra que los temas de evaluación y gestión del riesgo han sido trabajados desde la esfera gubernamental por las agencias de evaluación de tecnologías como la OTA en Estados Unidos. ${ }^{3}$ Desde el punto de vista filosófico, autores como Beck, Funtowicz, Ravetz, Shrader-Frechette y Wynne son algunos de los principales exponentes. El concepto de evaluación constructiva de tecnologías (ECT) ha sido desarrollado principalmente por grupos de investigación de la Universidad de Twente, liderados por sus principales exponentes Rip y Schot. Este concepto se relaciona, además, con la perspectiva construccionista de la tecnología cuyos autores son Bijker, Pinch y Hughes. Se mostrarán más adelante los enfoques y algunos de los debates asociados a cada una de estas líneas de trabajo.

En general, se puede observar que en Estados Unidos la preocupación se orienta hacia la gestión del riesgo, la evaluación de tecnología y la inclusión de la participación pública en la toma de decisiones, mientras que en el Reino Unido se trabaja el tema de la incertidumbre y la validación de la información científica para medir o evaluar los riesgos tecnológicos, así como la inclusión de la participación pública como estrategia para reducir esta incertidumbre. En el caso de los países bajos, se trabaja en la evaluación participativa de tecnologías como una forma de ejercer una injerencia en el cambio tecnológico.

3 Office of Technology Assessment. 
En relación con lo anterior, un tema transversal en estas líneas y, en general, en el estudio de las relaciones de ciencia, tecnología y sociedad, es la participación pública y el concepto de percepción pública. El tema de la percepción es utilizado para conocer las posturas de diferentes actores respecto a la ciencia y la tecnología y sus riesgos. Es un instrumento utilizado en la política tecnológica. En el caso de la participación pública, es utilizado en dos vías. La primera para legitimar algunas decisiones en ciencia y tecnología evitando de este modo la oposición de la población. En la segunda vía, como estrategia para la democratización de las decisiones en política y proyectos de carácter científico y tecnológico.

Finalmente, la educación ambiental es una apuesta de las naciones con el objetivo de contribuir al desarrollo sostenible y la preservación de los recursos naturales. En este sentido las Naciones Unidas han declarado la década de la educación para un futuro sostenible (2005-2014), con el propósito de "contribuir a la formación de ciudadanas y ciudadanos conscientes de los problemas socio-ambientales a los que se enfrentan y preparados para participar en la toma de decisiones fundamentadas" (Vilches, et al, 2009).

\section{Un panorama de la preocupación por el medio ambiente}

La forma de vida, la sobrevivencia, el sustento, las herramientas y las técnicas empleadas por una determinada sociedad van cambiando a medida que pasa el tiempo. Las sociedades primitivas, por ejemplo, basaban su sobrevivencia en una estrecha relación con la naturaleza, una relación de hermandad, convivencia y cooperación. Las herramientas rudimentarias facilitaban las actividades de caza y pesca para conseguir alimento y su gran fuente de energía era la fuerza muscular, el impacto ambiental era mínimo. Más adelante, al convertirse en sociedades sedentarias, las anteriores herramientas fueron reemplazadas por otras más sofisticadas que les permitían manejar la tierra y dedicarse a la agricultura, generándose así la denominada revolución agrícola. Se inició la gran modificación del ambiente: terrenos no sólo dedicados a la agricultura sino, también, a las construcciones para vivienda, se talaron extensas zonas de bosques y se erosionó el suelo. A medida que fue avanzando el tiempo, la forma de vida cambió, los grupos que conformaban las primeras sociedades eran cada vez más grandes, la organización social, las actividades y las técnicas empleadas para aprovechar los recursos naturales y garantizar su sobrevivencia se modificaban constantemente. La fuerza muscular, único recurso energético empleado en las primeras civilizaciones, fue reemplazada por nuevas fuentes de energía: los combustibles fósiles, y llegó la revolución industrial. Fue el comienzo de la utilización de los recursos no renovables. 
Posteriormente, empezó a utilizarse el ambiente sin respetar sus límites y tasas de recuperación. Hoy en día, las sociedades de consumo declaran al planeta en emergencia ${ }^{4}$ debido al uso irracional de los recursos: algunos recursos considerados renovables, como el agua, comienzan a considerarse como recursos de difícil renovación o recuperación.

Los actuales hábitos de consumo, las formas de producción industrial, los métodos de explotación de recursos, el aumento de los desechos, entre otros, ejercen una presión tan grande sobre los ecosistemas que superan su resiliencia o capacidad de recuperación. Cuando esta situación se prolonga, genera una crisis ambiental, repercutiendo en el sistema productivo arrastrando consigo la crisis energética y, siendo la energía el corazón del crecimiento económico, su escasez está relacionada directamente con crisis económicas de gran magnitud (Avellaneda, 2002). Esta situación de crisis ha generado un llamado de atención para todo el mundo. El planeta se encuentra al límite, con una restricción de los recursos y de la capacidad de absorción de desechos. Debe darse prioridad a las medidas que garanticen el mantenimiento de todos los ecosistemas sin sobrepasar los denominados límites de la tierra. Así los riesgos para los seres vivos disminuirán.

La incógnita, en la actualidad, es cuánto van a durar los recursos o cómo deben manejarse. Es por lo tanto un tema de interés general que ocupa a los especialistas que, con sus investigaciones, modelos de simulación y análisis de los cambios climáticos pretenden determinar la situación real del medio ambiente y las alternativas que existen para mitigar el deterioro del mismo. Hasta el ciudadano común, que trata de entender la situación, debe determinar cuál es su influencia y cómo puede participar para evitar la prolongación de la problemática ambiental. ¿Se necesitan únicamente nuevas tecnologías para minimizar el impacto ambiental y seguir cumpliendo con lo que ha originado todas estas modificaciones desde tiempos pasados hasta el momento: garantizar la sobrevivencia en la tierra?

Es así como el estudio del medio ambiente, la problemática existente y las soluciones para restablecer un equilibrio natural, no corresponden solamente a las ciencias naturales (Física, Química, Biología y Matemáticas) sino, también, a las ciencias humanas (Sociales, Económicas, Legales), las ingenierías y tecnologías, las ciencias de la salud y las ciencias de la educación. Cada una de ellas

4 Desde 1990 se empieza a hablar de "la emergencia planetaria", constituida por un grupo de problemas relacionados entre sí y que se potencian mutuamente. La contaminación que potencia el cambio climático y la degradación de los ecosistemas. El agotamiento de los recursos potenciado por la explosión demográfica, lo que a su vez produce desequilibrios insostenibles y la pérdida, entre otras cosas, de diversidad biológica y cultural (Vilches, et al., 2009). 
constituye parte esencial para estudiar todo lo relacionado con el medio ambiente, contribuir a su conservación y perpetuidad para el bien de la humanidad, al igual que determinar opciones para detener la llamada "emergencia planetaria" (Vilches y Gil, 2003).

Uno de los problemas relacionados con el medio ambiente y que actualmente preocupa a la humanidad es el cambio del clima global, el aumento de la temperatura y sus consecuencias sobre las diferentes formas de vida. Se realizan diferentes esfuerzos para detener este calentamiento global, como la convención de cambio climático incluida en la declaración de la conferencia de las Naciones Unidas sobre medio ambiente y desarrollo (1992) en Río de Janeiro, con el fin de detener este proceso. ¿Hasta qué punto la convención se ajusta a ese objetivo? ¿Qué se puede esperar de los procesos de negociación que se realicen ahora y en los próximos años para acordar los instrumentos requeridos para la convención y que quedaran consignados en los denominados protocolos? Se ha pasado por el protocolo de Kyoto, la cumbre de Copenhague, pero siempre queda faltando algo. Si los dirigentes políticos pudiesen confiar en que el cambio es posible, quizá llegasen a un acuerdo histórico (Jacobson y Deluchi, 2010).

¿Cómo controlar las emisiones generadas por el uso de combustibles fósiles y que incrementan la concentración de gases de invernadero? Según un artículo escrito por Jacobson y Deluchi (2010) sobre la energía sostenible, es de capital importancia la creación de un acuerdo para asegurar la reducción de las emisiones de gases de efecto invernadero en las próximas décadas; en este sentido la medida más efectiva para fijar ese objetivo sería sustituir, en gran medida, los combustibles fósiles por unas fuentes de energías limpias y renovables, sería hacer más sostenible la vida humana en la tierra.

Desde hace al menos 10 años se han venido analizando distintos componentes del problema. Hace muy poco, un estudio de la Universidad de Stanford realizado por Jacobson y Deluchi (2009) clasificaba los sistemas de energía según sus repercusiones en el calentamiento global, la contaminación, la agricultura, la flora y la fauna y otras áreas objeto de preocupación. Las mejores opciones fueron la energía eólica, la solar, la geotérmica, la mareal y la hidroeléctrica, basadas, respectivamente en el viento, luz solar, el calor interno de la tierra, la fuerza mareal y los saltos de agua. La implementación de políticas apropiadas para establecer mercados del carbono que funcionen bien e impulsar la investigación, el desarrollo y la difusión de tecnologías es necesaria para proporcionar incentivos y propiciar las reacciones de mitigación por parte de hogares y empresas (Stern, 2007). Es importante recordar que el cambio climático es un cambio global, los países no deben actuar individualmente, se requieren acciones conjuntas entre 
naciones, basadas en acuerdos o marcos internacionales, propiciar la cooperación tecnológica entre naciones y la inversión destinada al desarrollo de nuevas tecnologías, que mejoren la eficiencia energética.

Actualmente, las sociedades se esfuerzan por mantener el equilibrio de la naturaleza. ¿Cómo lograr un equilibrio en el ambiente? ¿Cómo lograr que el ciudadano común, los gobernantes, las empresas se sientan involucrados? Surge como posibilidad el desarrollo sostenible, considerado el paradigma de desarrollo idóneo del siglo XXI y donde se reconoce a la sostenibilidad como revolución cultural, tecnocientífica y política.

Este paradigma implicaría el desarrollo de tecnologías que permitieran explotar los recursos naturales con el mínimo impacto ambiental, reformas sociales y económicas hacia la equidad, así como profundos cambios en el comportamiento de la población en relación con el uso de energía y el consumo de bienes y servicios. Se dirigen muchos de los esfuerzos de investigación hacia el logro de tecnologías favorecedoras de un desarrollo sostenible: nuevas fuentes de energía -eólica, solar, geotérmica-; incremento en la eficiencia de la obtención de alimentos -optimización del uso del suelo, ingeniería genética-; aplicación de la biotecnología; prevención de enfermedades y catástrofes; tratamiento de residuos y educación para la sostenibilidad.

La aplicación de las nuevas tecnologías genera en algunas ocasiones incertidumbre por parte de los innovadores: costos, eficiencia, efectos secundarios y economía. Para garantizar el apropiado uso de las innovaciones tecnológicas y promover la confianza de quienes las utilizarían, se creó el Principio de Precaución, conocido también como de cautela o prudencia (Vilches, et al., 2009). Básicamente se refiere a la investigación exhaustiva de las repercusiones posibles de una determinada tecnología antes de implementarla.

A este respecto, las "Pautas para aplicar el principio de precaución a la conservación de la biodiversidad y la gestión de los recursos naturales", diseńadas por The Precautionary Principle Project, en el que ha trabajado un amplio grupo de expertos de diferentes campos, regiones y perspectivas; han introducido -aunque tan sólo están vigentes en algunos países-instrumentos como la Evaluación del Impacto Ambiental (EIA), para prevenir los impactos ambientales de las tecnologías que se proponen, analizar los riesgos y facilitar la toma de decisiones para su aprobación o no, así como las Auditorías Medioambientales (AMA) de las tecnologías en funcionamiento para conocer la calidad de sus productos o de sus prestaciones.

El objetivo es superar la búsqueda de beneficios particulares a corto plazo que ha caracterizado, a menudo, el desarrollo tecnocientífico, y potenciar tecnologías 
básicas susceptibles de favorecer un desarrollo sostenible que tenga en cuenta, a la vez, la dimensión local y global de los problemas a los que se enfrentan las sociedades actuales.

Además, es necesario señalar que ya existen soluciones científico-tecnológicas para algunos de los problemas actuales: las prácticas agrícolas y el uso de la tierra, la disminución de la pérdida de biodiversidad, el uso racional del agua y la contaminación de fuentes hídricas -aunque, naturalmente, será siempre necesario seguir investigando- pero estas soluciones tropiezan con las barreras que suponen los intereses particulares o las desigualdades en el acceso a los avances tecnológicos, que se acrecientan cada día. Estos factores también hacen parte de la evaluación tecnológica.

Por último, la institucionalización de los problemas medioambientales, se da a partir de las conferencias organizadas por las Naciones Unidas, dando lugar a la creación de políticas de carácter global sobre el medio ambiente con base en la cooperación internacional. Sin embargo, el conflicto de intereses entre naciones y, a su vez, entre actores sociales y del Estado, no ha permitido que esta institucionalización haya tenido el impacto deseado (Oltra, 2006).

\section{Áreas de trabajo en las relaciones CTS y el medio ambiente}

En el tema del medio ambiente, la ciencia y la tecnología han jugado un doble papel en la forma de abordarlo. En primer lugar, se ha culpado a la ciencia y a la tecnología de provocar la mayor parte de los problemas que actualmente aquejan al planeta; $y$, en segundo lugar, se le otorga la responsabilidad de crear soluciones para estos problemas, a partir de la creación de nuevas tecnologías, del cambio en los patrones de consumo y de la exploración de alternativas para la satisfacción de las necesidades de las personas. En este sentido, se producen dos perspectivas teóricas desde lo que Oltra (2006) denomina sociología ambiental, estas son: la sociedad del riesgo y la modernización ecológica.

En la primera perspectiva el análisis de la crisis ecológica ${ }^{5}$ se plantea de forma reflexiva al considerar de manera fáctica que los problemas medioambientales son reales y de carácter global y, de forma construccionista, al plantear que los peligros son construidos socialmente por los actores sociales. Es así como nace la definición de riesgo en tanto que se interpreta como los peligros que dependen de decisiones humanas. ${ }^{6}$ Dicho de otro modo, son impactos negativos que no

5 Es preciso señalar que, si bien la actividad humana ha tenido un impacto en su entorno, como indica Lobera (2008), sólo en la actualidad este impacto ya no es de carácter local, sino que se extiende a nivel global en términos de espacio y además sostenido en el tiempo, lo que implica que afecta a todos los habitantes del planeta y a las generaciones futuras.

6 El concepto de riesgo es institucionalizado por Ulrich Beck en su obra de 1986 La Sociedad del Riesgo. 
están por completo determinados y, por tanto, se requiere de la asignación de probabilidades a los mismos. En esta vía se encuentra el enfoque de gestión del riesgo, la evaluación tradicional de tecnologías y la percepción del riesgo.

En la segunda perspectiva, se plantea que la crisis es un proceso intermedio hacia el cambio, mediado por la negociación en los aspectos sociales, económicos, políticos, para la ejecución de alternativas que permitan avanzar hacia la sostenibilidad. En este sentido, la sociedad avanzaría hacia un cambio en las instituciones, la consolidación de políticas medioambientales y la modificación de los patrones de consumo y estilos de vida, entre otros, que le permita a la sociedad llegar a la modernización ecológica (Oltra, 2006). En la perspectiva de modernización ecológica, la ciencia y la tecnología juegan un papel importante en la transformación de los procesos de producción y consumo, así como en la creación de innovaciones tecnológicas ambientales. Es decir, que la ciencia y la tecnología junto al estudio de la economía, el Estado y la movilización social, cobran importancia en la prevención y solución de problemas ambientales, y no sólo en su creación (Oltra, 2006). En este sentido, esta perspectiva tiene una alta relación con la evaluación constructiva de tecnologías y la participación pública en temas tecnocientíficos.

En resumen, las acciones emprendidas bajo la perspectiva de la crisis ecológica buscan realizar la medición de los impactos, reducir los riesgos y gestionarlos de la mejor forma para ayudar a reducir su presión sobre el medio ambiente y las sociedades. Mientras que bajo la perspectiva de la modernización ecológica las herramientas conceptuales se orientan a cambios en la trayectoria de las tecnologías, en las instituciones y en la manera en que las sociedades se conciben en relación con el medio ambiente. No es el objeto de este trabajo establecer cuál de las dos perspectivas es la adecuada en las sociedades latinoamericanas. Se enuncian en este texto para delimitar los supuestos bajo los cuales se aplican los conceptos que se desarrollan a continuación.

\section{Gestión del riesgo}

Cuando se habla de riesgo, es preciso distinguir la diferencia entre peligro y riesgo. En el peligro el ser humano no tiene control sobre el mismo pues éste es ocasionado por la naturaleza, y en el pasado por los dioses o el destino. En cambio, los riesgos son aquellos peligros que pueden ser causados o prevenidos por acciones y decisiones humanas (Renn, et al., 1995). A nivel internacional se ha entendido que la ciencia y la tecnología impactan en diversos sentidos y dimensiones sociales. Con un factor adicional, y es que este impacto no siempre es positivo. En este sentido, se puede hablar de un riesgo asociado a la tecnología. Esta situación ha incrementado la sensibilización social hacia temas relaciona- 
dos con políticas de innovación tecnológica, gestión ambiental, planificación urbana, etc., permitiendo que se creen organizaciones y asociaciones que han empezado a tener injerencia, ya sea por presiones o por negociaciones, en el ámbito político y social.

Por ejemplo, uno de los principales problemas que preocupa en la actualidad es el cambio climático y sus efectos: inundaciones o sequías extremas, extinción de especies, deshielo de los glaciares, aumento en el nivel del mar, entre otros. Donde generalmente, las poblaciones más pobres son las más vulnerables y las que se encuentran en mayor riesgo de sufrir las repercusiones de esta situación. Desde el punto de vista político la preocupación en estos temas se centra en cómo manejar la incertidumbre científica y cómo actuar frente a los riesgos (Antal, 2007).

Pero evaluar los riesgos no es suficiente, como afirma Beck en una crítica a la tecnocracia (2006): "la ciencia fija los riesgos y la población percibe los riesgos". Como consecuencia, el debate se ubica en la credibilidad de los hechos experimentales, de las pruebas técnicas y en la complejidad de la racionalidad científica y la racionalidad social. En términos generales, esta complejidad se presenta debido a que la base científica de los riesgos se centra en la objetividad de las afirmaciones en un marco de probabilidades, y en juzgar si estas probabilidades son altas o bajas dependiendo de intereses sociales. En palabras de Beck (2006), una probabilidad de un accidente catastrófico, aunque sea mínima, es inaceptable para los afectados, para quienes esta catástrofe puede significar el exterminio.

Otra situación donde se enfrentan la racionalidad científica frente a la social, es aquella donde los actores sociales evidencian riesgos que no son aceptados por los científicos, poniendo en duda la legitimidad de las pruebas y los hechos que la soportan. Es así como aparece con el concepto del riesgo, el concepto de la incertidumbre ligado a la toma de decisiones, sobre todo en el ámbito político, con base en información científica fiable de los impactos y riesgos tecnológicos. En este sentido, una de las críticas más fuertes a la evaluación de riesgos se refiere a que implícitamente traen juicios de valor, lo que trae consigo inferencias subjetivas y, por lo tanto, una evaluación engañosa y en la mayoría de los casos inequitativa a la hora de compartir los riesgos (Shrader-Frechette, 1997). ${ }^{8}$ Es el caso de Estados Unidos, que debido a sus intereses económicos, niega las pruebas científicas acerca de las actividades antropogénicas como causa del calentamiento global. O el caso de Canadá, que en su apuesta hacia la competitividad utilizando

7 También se refiere, en su obra, a los riesgos de clase, la globalización de los riesgos.

8 Según Durbin (1995) Shrader-Frechette es la autora más reconocida en trabajar los problemas y las falacias en la evaluación riesgo/costo/beneficio y una de las filósofas con un mayor trabajo en la evaluación de tecnologías en Estados Unidos. 
la biotecnología, rechaza la evidencia acerca de que hay factores diferentes a las pruebas científicas que, desde la percepción pública, llevan a evitar el consumo de alimentos provenientes de organismos genéticamente modificados (Antal, 2007). ${ }^{9}$

Para mediar en este conflicto -y ante la necesidad de que la ciencia y la tecnología, que han ayudado al progreso, también contribuyan a disminuir los riesgos que la industrialización ha producido- Funtowicz y Ravetz (1997) proponen lo que ellos denominan Ciencia Post-Normal. Ésta se basa en que la calidad de la investigación científica sea valorada no sólo por expertos sino por comunidades de evaluadores extendidas. Esto debido a dos razones: la primera es la existencia de incertidumbre en un sistema complejo como el medio ambiente, cuyos problemas pueden abordarse de distintas maneras y que conlleva la inclusión de valores en el tratamiento de los mismos. La segunda es la calidad que se le debe otorgar a la información científica en la toma de decisiones tecnológicas. En consecuencia, su propuesta apunta hacia la democratización de la ciencia, con un nuevo modelo de valoración científico-tecnológico que cree espacios para la participación pública, donde la ciencia aporta significativamente a la solución de problemas pero en un contexto de incertidumbre de los sistemas medioambientales y la relevancia de los valores sociales (Funtowicz y Ravetz, 1997).

Ahora bien, cuando las pruebas parecen no ser suficientes, pero existe una duda razonable de los posibles riesgos de una acción, como es el caso de la implementación de tecnologías en el impacto medioambiental, se ha propuesto, como guía para el establecimiento de políticas públicas en temas del medio ambiente, seguir el principio de precaución. Este principio mencionado por Wynne (1997), Carr e Ibarra (2004) y Luján y López (2004), y formulado originalmente en la Declaración de Río, en la Conferencia de las Naciones Unidas sobre Medio Ambiente y Desarrollo de 1992, afirma que ante la posibilidad de un dańo grave o irreversible, la falta de certeza científica o de pruebas contundentes, no debe ser un obstáculo para tomar medidas para evitar el daño. Sin embargo, la aplicación de este principio también ha generado propuestas en dos vías: una más radical, propone que se utilice el principio de precaución como criterio para la selección de tecnologías, y la otra como principio para el diseño de políticas públicas. Un ejemplo de la aplicación de este principio es el caso de los alimentos transgénicos. Estos han sido fuertemente cuestionados por diferentes sectores sin una evidencia clara de productos comerciales que hayan tenido un efecto negativo sobre la salud. Aquí la preocupación es, desde el punto de vista teórico, por los

9 Una revisión sobre las controversias sociales, políticas y científicas en el tema del cambio climático y los organismos genéticamente modificados se encuentra en Antal (2007). 
posibles efectos negativos que no puedan ser probados mediante la ciencia y la tecnología actual (Palou, 2004).

La promoción de este principio, en conjunto con tecnologías favorecedoras de la sostenibilidad, ha sido propuesta por Vilches, et al., (2009) como un compromiso de los educadores en la década de la educación para el desarrollo sostenible, ${ }^{10}$ debido a que este principio puede interpretarse como un enlace entre la gobernabilidad y el riesgo (De Morchi y Funtowicz, 2004). Sin embargo, también ha sido criticado por algunos autores al considerar que este principio se convierte en una excusa para evitar la implementación de ciertas tecnologías. Las preguntas sobre las que se basa la crítica son: ¿̨cuál debe ser la norma o estándar usado para medir el daño?, ¿qué tipo de riesgos son aceptados? Y, por último, ¿qué clase de medidas de precaución deben ser tomadas? (Ahteensuu, 2004).

\section{Evaluación constructiva de tecnologías (ECT)}

La preocupación por la problemática social y ambiental que la innovación tecnológica produjo en décadas pasadas, motivó un cambio de actitud respecto a la introducción de tecnologías sin una evaluación de los efectos que traen consigo (Cutcliffe, et al., 1992).

Inicialmente la evaluación de tecnologías se basó en el conocimiento de expertos acerca de la interacción de las tecnologías, la naturaleza y la sociedad, revisando los impactos sociales y ambientales que en la mayoría de los casos, se reducía a valoraciones económicas de costo-beneficio. ${ }^{11}$ Es así como la evaluación constructiva de tecnologías nace ante la crítica al modelo tradicional por su inoperancia y por la presión social, debido a que la percepción y la evaluación social de los riesgos diferían de los informes de los expertos, ya que cada actor podía percibir distintos tipos de riesgo. Por lo tanto, la evaluación constructiva de tecnologías es una respuesta a la necesidad de que los ciudadanos y los diferentes actores tengan una mayor implicación en la gestión del cambio tecnológico. Además, los estudios con una perspectiva social y cultural pueden ayudar a entender los complejos procesos involucrados en el cambio tecnológico y brindar estrategias o pautas hacia una mayor sostenibilidad (Jamison y Rohracher, 2001).

Debido a que la ECT propone que se amplíe el diseño de las tecnologías, reuniendo a todas las partes interesadas desde el principio y durante todo el proceso de diseño, ésta ayuda a entender y a introducir formas de participación en el

10 La década de educación para un futuro sostenible (2005-2014) que promueve las Naciones Unidas, tiene como objetivo principal formar ciudadanos y ciudadanas conscientes de los problemas relacionados con el medio ambiente, y formados para participar en la toma de decisiones necesarias para su solución.

11 Una crítica en este tipo de valoraciones es: cómo otorgar valores económicos a bienes ambientales en el presente y su importancia para generaciones futuras. 
diseño de tecnologías. Es decir, que se realiza la inclusión de la participación no sólo en la selección de tecnologías, sino en el diseño de las mismas y se incluyen además las cuestiones éticas y culturales que permitan la aceptación de este tipo de tecnologías (Schot, 2001; Jamison y Rohracher, 2001).

Específicamente, en el campo ambiental, es una estrategia que ha cobrado relevancia debido a la creciente preocupación por los altos niveles de contaminación en el agua, el suelo y el aire que producen ciertas tecnologías. Los casos comunes en el área de desarrollo sostenible son: tecnologías limpias, movilidad sostenible, energía renovable y arquitectura sostenible (Schot, 2001; Jamison y Rohracher, 2001). Adicionalmente también se realizan evaluaciones de esta naturaleza en el caso de la utilización de productos transgénicos, en la producción agrícola y de la biotecnología en las diferentes aplicaciones industriales. En general, esta metodología ha sido aplicada por organizaciones de política pública en Dinamarca y los Países Bajos, la Unión Europea y organizaciones como la OECD (Genus y Coles, 2005; Schot, 2001).

Según Genus y Coles (2005) la ECT parecería inspirarse en la necesidad de resolver la dicotomía entre la promoción de la tecnología y su control (manejo de consecuencias). Aquí el aprendizaje sobre los posibles impactos y sobre las opciones que puede tomar una tecnología se dan ex ante. En este sentido la ECT tiene tres meta-criterios: anticipación, reflexividad y aprendizaje social. Los cuales funcionan para construir y para evaluar los procesos de ECT (Schot, 2001). Es decir, se busca la anticipación de impactos de la tecnología más que atribuir la responsabilidad sobre los problemas asociados con el desarrollo (Genus y Coles, 2005). El aprendizaje social necesario para aclarar los valores fundamentales implícitos en el diseño y valoración de la tecnología. Todo esto basado en la capacidad de los actores - reflexividad- de considerar el diseño de la tecnología y el diseño social como un proceso integrado en el cual se negocian los conceptos del diseño a partir de las expectativas de los actores, los contextos sociales y los criterios técnicos (Genus y Coles, 2005; Schot, 2001).

Un supuesto de la ECT es que la trayectoria de la misma puede ser modificada. En el caso de los modelos evolucionistas, el desarrollo tecnológico se da por selección y variación. La selección tiene que ver con la ejecución y evaluación de diferentes opciones, las cuales se presentan a ensayo y error. La variación tiene que ver con las condiciones contextuales en que se desarrollan el cambio tecnológico, ${ }^{12}$ ya sea ex post por presiones del mercado, o ex ante debido a que

12 Por ejemplo, en el caso de las energías renovables, se tienen tecnologías como la energía eólica o la solar desarrolladas hace bastante tiempo. Sin embargo, por presiones del mercado, a través de políticas e intereses económicos por el petróleo no se han difundido y, por lo tanto, no se ha dado de forma contundente un cambio tecnológico hacia tecnologías más democráticas y más amigables con el medio ambiente. 
algunos actores, por ejemplo las empresas, pueden anticipar los cambios, al relacionar las oportunidades técnicas y de mercado (Schot, 1997).

Schot (1997) muestra la aplicación de la ECT apoyado por los conceptos de selección y variación de la teoría evolucionista, en el caso de las tecnologías limpias, ${ }^{13}$ en el sentido en que diferentes grupos de actores podrían presionar la trayectoria tecnológica hacia la utilización de tecnologías limpias, mediante tres estrategias: el desarrollo de variaciones alternativas, la modificación del ambiente de selección y la creación o utilización de nexos tecnológicos. ${ }^{14}$ En la primera estrategia se trata de fomentar la producción de tecnologías alternativas, por ejemplo, la energía eólica o la energía solar. Sin embargo, no es suficiente con que existan tecnologías alternativas, pues se presenta, en la mayoría de los casos, un problema de comercialización y difusión de la misma, debido a que el mercado no es receptivo. En esta medida, no basta con hacer las variaciones sino que el ambiente de selección debe tener cambios para el éxito de la implementación de tecnologías alternativas.

La segunda estrategia surge como complemento a esta situación, y consiste en modificar el ambiente de selección para presionar el cambio tecnológico. Esta presión puede provenir del gobierno, a través de políticas y estándares altos en cuanto a la prevención de la contaminación. Así como restricciones fuertes a la utilización de tecnologías tradicionales que generen impactos negativos al medio ambiente. También las aseguradoras pueden explorar el mercado de riesgos ambientales y, una vez evaluados, pueden exigir a las empresas la implementación de acciones preventivas o de tecnologías limpias como condición para tomar el seguro. Aquí, el gobierno debería exigir la consecución de un seguro, para ejercer una presión más efectiva. Otros actores que pueden presionar para cambiar el ambiente de selección son las asociaciones de fabricantes, el eslabón proveedor -usuarios y consumidores, al modificar los patrones de consumo y exigir etiquetas ecológicas que les garanticen que el producto consumido sea amigable con el medio ambiente.

Por último, la utilización de nexos tecnológicos como estrategia para el cambio hacia la utilización de tecnologías limpias se enfoca en los departamentos de

13 En el caso de la contaminación y degradación ambiental y su relación con la producción industrial, se debe distinguir entre tecnologías de final de proceso, que son aquellas que aparecen para disponer los residuos o desechos que resultan del proceso de producción y que intentan mitigar la contaminación; y aquellas llamadas tecnologías limpias que pretenden modificar el proceso mismo de producción y evitar la aparición de desechos contaminantes o la depredación de recursos que deterioren el medio ambiente.

14 El nexo tecnológico se refiere a los actores "que traducen las demandas realizadas desde el ambiente en recomendaciones u objetivos de desarrollo tecnológico y, a la vez, imponen al ambiente nuevas variaciones tecnológicas" (Schot, 1997). 
control de calidad, de mercadeo, y otros departamentos sensibles a la presión de actores externos como el público o la cadena proveedor-usuario.

Una crítica al ECT, es que reduce las condiciones de producción de la tecnología a sus circunstancias de recepción y uso (Lawler, 2003), además supone que estas circunstancias son construidas socialmente. Es decir, que una crítica sería su posición hacia el determinismo social de la tecnología. ${ }^{15}$ Así mismo, Genus y Coles (2005) plantean una crítica a la ECT desde dos perspectivas: la primera de ellas enfatiza que existen factores estructurales, como los desequilibrios de poder entre los actores y las estructuras de gobierno, que afectan el rol de los grupos o ciudadanos individuales dispuestos a jugar en el desarrollo tecnológico. En la segunda perspectiva se analizan los aspectos culturales, comunicacionales y discursivos en la valoración de tecnologías. Esto es, que se cuestiona si todos los grupos involucrados tienen el acceso a las habilidades discursivas y comunicativas ya que, de otro modo se podrían presentar inequidades en el acceso al debate y la toma de decisiones.

Algunos dilemas a los que se enfrenta la ECT son: a) insuficiente participación, lo cual tiene el riesgo de perder la legitimidad de procesos que requieren gran esfuerzo económico por parte de las autoridades gubernamentales; b) procesos lentos, o debates "interminables" dada la imposibilidad de consenso entre las partes debido a posturas irreconciliables.

En la práctica se plantea el desafío de cómo ser eficiente, a la vez de tener los tiempos suficientes para la toma de decisiones; y cómo involucrar los grupos de interés a través de una muestra de ciudadanos. LA ECT depende, entre otras cosas, de qué tan involucradas estén las sociedades respecto a apreciar y crear espacios para la negociación y el criticismo socio-técnico.

\section{Participación pública y percepción pública}

El tema de la participación pública en asuntos tecnocientíficos, tiene su origen en dos situaciones. En la primera, se considera que la participación pública surgió de la percepción de los efectos negativos de algunas de las políticas de innovación tecnológica y de las amenazas medioambientales (Doelle y Sinclair, 2006). En este aspecto las instituciones y los gobiernos empezaron a incluir la percepción del público en la determinación de políticas en temas como la salud y el medio ambiente. La segunda situación, se relaciona con el fracaso de la transferencia de tecnología hacia los países en vías de desarrollo, lo que llevó a establecer que la práctica tecnológica implica no sólo el diseño y la implementación sino,

15 El determinismo social de la tecnología plantea que las dinámicas tecnológicas son el resultado de las interacciones sociales y la percepción de los grupos de actores. En contraposición se encuentra el determinismo tecnológico que plantea que el desarrollo de la tecnología es el resultado sólo de la dinámica interna de la misma. 
también, la operación y el mantenimiento de esta tecnología enmarcada en los valores sociales y culturales de la comunidad beneficiada. La legitimación de la introducción de nuevas tecnologías no es per se, sino que cobra importancia sus impactos sociales y medioambientales (López, et al, 1998). En este sentido la participación pública es una característica de sociedades más democráticas (Doelle y Sinclair, 2006).

En el área del medio ambiente la participación pública se ha orientado hacia la toma de decisiones concernientes a la política pública, que involucra proyectos de carácter ambiental tan controvertidos como el uso de la biotecnología en la producción de alimentos, el cambio climático, y la introducción de tecnologías alternativas para la generación de energía y para la producción más limpia. Los métodos participativos han sido utilizados en la valoración de tecnologías como una forma de mejorar esta evaluación en los aspectos éticos y sociales, y democratizar la toma de decisiones en los temas científico-tecnológicos. Es decir, se requiere la participación para garantizar la gobernabilidad, la sostenibilidad y la legitimación de la tecnología. Así mismo, el fomento y la sensibilización por la participación de los actores sociales en el ámbito público es necesaria para fortalecer otras áreas de trabajo del campo CTS en lo ambiental como lo son la evaluación constructiva de tecnologías y la gestión del riesgo.

Esta implicación del público se ha realizado de variadas formas, como son: distribución de información a un público objetivo, selección de una parte de la población para conocer su opinión a través de grupos focales, encuestas o ejercicios consultivos. Otras formas de implicación están relacionadas con ejercicios en los cuales el público - población o comunidad- tiene un grado de autoridad en la toma de decisiones (Rowe y Frewer, 2000). En cuanto a los motivos o argumentos para la participación, Fiorino (1990) presenta tres razones: La participación efectiva de los no expertos en la toma de decisiones ayuda a legitimar y a obtener mejores resultados en las mismas. La segunda razón es que la orientación tecnócrata es incompatible con la democracia al ignorar a los ciudadanos. Este argumento acepta como supuesto "ético" que son los ciudadanos los que pueden juzgar mejor sus intereses. Y, por último, los juicios de los no expertos son tan válidos y pertinentes como los de los expertos. Fiorino (1990) afirma que los juicios de los no expertos revelan valores sociales y políticos que los modelos de los expertos desconocen.

La participación tiene un alto significado en la democracia y por lo tanto debe ser efectiva desde el punto de vista del proceso y el resultado. Sin embargo, según King et. al. (1998), la verdadera participación va más allá de encontrar erramientas y técnicas para involucrar a la población en las decisiones públicas. 
La autentica participación es aquella que trabaja por todas las partes interesadas y a la vez estimula el interés y el esfuerzo tanto del estado (gobernantes) como de los ciudadanos

En cuanto al fortalecimiento de la participación pública, se plantea la alfabetización científica y tecnológica de los ciudadanos, con el objetivo de promover la participación pública en temas medioambiéntales y en la valoración de proyectos científico-tecnológicos que generan riesgos en el medio ambiente y la salud humana.

Mientras la participación pública aparece como una serie de estrategias por las que gobiernos y movimientos sociales propenden por una mayor participación de la sociedad en general en asuntos de ciencia y tecnología, la percepción pública remite más bien a las preocupaciones que circulan en la sociedad ante el problema medioambiental. En el caso de la percepción del riesgo, cada grupo de actores percibe de diferentes formas los riesgos o situaciones. Es decir, que la percepción del riesgo está más asociada a factores socioculturales y a valores morales, que a una objetividad del fenómeno y una única realidad (Suárez, et al., 2004) Ambas, sin embargo, se relacionan con la pregunta sobre el modo en que la sociedad debe hacer uso de los recursos, de tal forma que se contribuya al bienestar de las personas.

En conclusión, el fomento de métodos participativos, la evaluación de los mismos y la promoción de la participación de diferentes actores en la toma de decisiones permite fortalecer la aplicación de métodos como la gestión del riesgo y la evaluación constructiva de tecnologías.

\section{Educación ambiental}

Ciencia, Tecnología y Sociedad es un campo de estudio que se está expandiendo con rapidez en instituciones educativas de todos los niveles en los Estados Unidos, Canadá, Europa occidental, Australia, Nueva Zelanda y Sudamérica. Generado por la preocupación sobre las armas nucleares, el movimiento ecológico y la crítica tecnológica, el movimiento CTS surgió en los campus universitarios en los años sesenta y setenta (Cutcliffe, 1992), y se extendió en la educación secundaria en los años ochenta.

Desde sus propios orígenes, la universidad es sinónimo de pensamiento universal, el campus universitario es el espacio del encuentro con el saber y la creación y la recreación del mismo. Las preocupaciones sociales desde el propio nacimiento y desarrollo de las universidades han encontrado eco en el ámbito académico. La historia reciente ha tenido por lo menos dos claros ejemplos del papel de la universidad en las grandes transformaciones sociales: la Reforma de Córdoba en Argentina de 1918 que ha repercutido en la formación de las uni- 
versidades latinoamericanas desde entonces y la Revolución de Mayo de 1968 en Francia, que definió el carácter de la insurgencia juvenil, cuyas repercusiones aún se sienten a nivel global.

Fue también el movimiento universitario de 1968 en México, el que abrió espacios para el debate temprano sobre temas ambientales que se ha dado en la UNAM y en el Colegio de México, así como el movimiento estudiantil colombiano de 1970 y 1975 en Colombia, en el cual muchos de los actuales ambientalistas colombianos alimentaron su saber y generaron las primeras inquietudes ecológicas en los movimientos por la defensa del Parque Nacional Natural del Tayrona (Avellaneda, 2002).

En cada uno de los sitios donde es importante el conocimiento y, cuando se hace referencia a la problemática ambiental, cada centro educativo tendrá sus estrategias para lograr el desarrollo de este aprendizaje del saber ambiental. En la Figura 1 se pueden observar las condiciones de irrupción, asimilación, legitimación, y transmisión del saber ambiental en una organización disciplinaria del conocimiento, las estructuras sectorizadas del sistema de educación de cada universidad y lo que corresponde a cada una de ellas, como el contexto geográfico, social, político y cultural.

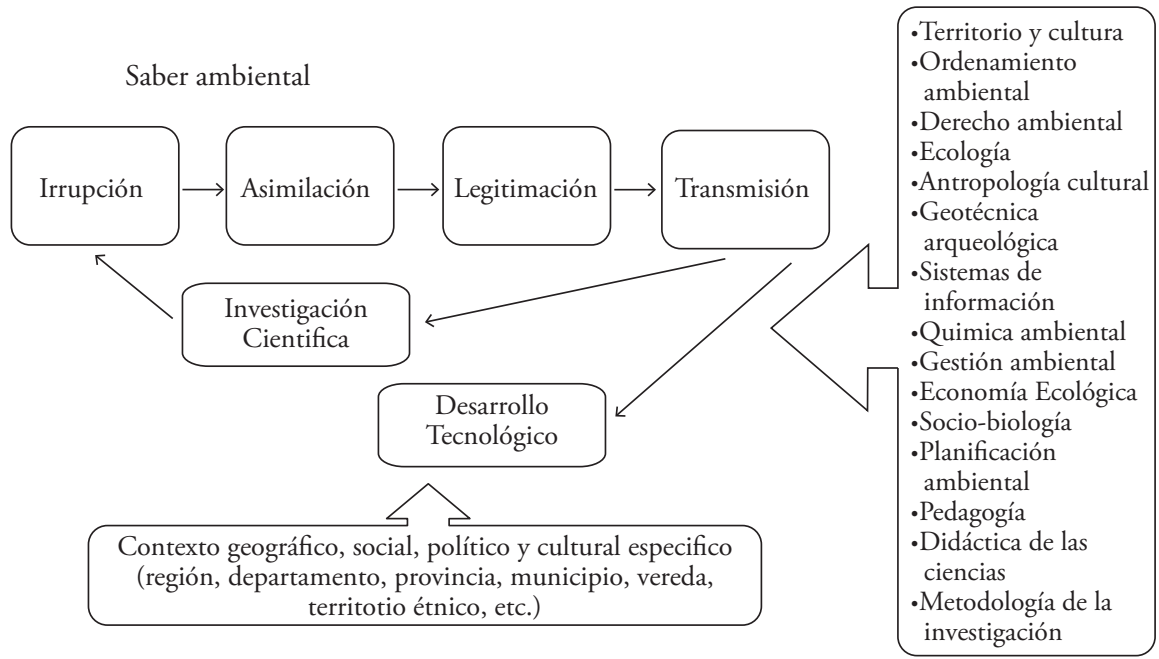

\section{Figura 1 Formación ambiental universitaria}

Fuente: Avellaneda (2002)

La investigación científica y el desarrollo tecnológico realizados en cada uno de los centros educativos, ejercen su acción sobre cada una de las condiciones que enmarcan el saber. Todo esto, relacionado con las realidades ambientales: 
territorio y cultura, ordenamiento ambiental y gestión ambiental, entre otras, las cuales variarán de acuerdo al momento en que esté enmarcada esta problemática ambiental.

Los aspectos ambientales han venido abriéndose paso en la sociedad durante el último decenio como una crítica al estilo de desarrollo y en la búsqueda de mejorar la calidad de vida de la población actual y las futuras generaciones. En este sentido, la universidad como órgano social de utilidad colectiva debe desempeñar un papel importante en el desarrollo del saber ambiental.

La organización curricular CTS comprende, generalmente, cinco fases las cuales también son llamadas "ciclo de responsabilidad" (Waks, 1990):

1. Formación de actitudes de responsabilidad personal en relación con el ambiente natural y la calidad de vida.

2. Toma de conciencia en investigación de temas CTS específicos y los efectos de distintas opciones tecnológicas sobre el bienestar de los individuos y el bien común.

3. Toma de decisiones con base en las opciones tecnológicas y teniendo en cuenta factores científicos, técnicos, éticos, económicos y políticos.

4. Acción individual y social responsable.

5. Generalización de la naturaleza sistémica de la tecnología y sus impactos sociales y ambientales, formulación de políticas en las democracias tecnológicas modernas y los principios éticos que pueden guiar el estilo de vida.

La educación es parte fundamental para entender la relación CTS. Una relación que ha variado a lo largo del tiempo y que sin duda, seguirá cambiando porque la investigación y la aplicación tecnológica no se detienen y, mucho menos, la utilización que hace la sociedad de los desarrollos científico-tecnológicos. La educación CTS debe ser integral y crítica, sin entrar en alguno de los extremos que ha caracterizado la relación entre sociedad y tecnología. En el primer extremo algunos consideran a la ciencia y la tecnología como los causantes de las peores situaciones en el mundo -estableciendo los grupos llamados tecnófobos-; mientras que el otro extremo ve a la ciencia y la tecnología como los salvadores de la humanidad -hablando de este modo de tecno-filia. Según Peña (1990) una educación en tecnología y sociedad adquiere sentido si:

1. Cuestiona las formas heredadas de estudiar y actuar sobre la naturaleza y la sociedad, reflejadas en la legitimación que se hace, a través del sistema educativo, de ciertas formas de conocimiento. 
2. Cuestiona la distinción convencional entre conocimiento teórico y conocimiento práctico, así como su distribución social entre los que piensan y los que ejecutan, que se refleja, a su vez, en un sistema educativo dual que diferencia entre la educación general y la vocacional.

3. Combate efectivamente la segmentación del conocimiento en todos los niveles de educación.

4. Promueve una auténtica democratización del conocimiento científico y tecnológico, de manera que éste no solo difunda, sino que se integre en la actividad productiva de las comunidades de manera selectiva, crítica y creativa.

Una educación en CTS va más allá de la evaluación de los impactos del desarrollo tecnológico y de la promoción de mecanismos que minimicen los efectos negativos. Promueve formas distintas de investigación y la transformación de los valores culturales asociados con la manera como el conocimiento científico y tecnológico se produce, se distribuye y se utiliza en una determinada sociedad.

Es necesaria la identificación y el estudio de problemas ecológicos específicos de la región para promover una conciencia ecológica que contribuya a su vez a generar la política tecnológica. Es importante recordar, que los cambios culturales pueden ser originados por los adelantos científico-técnicos y sus efectos inciden directamente en la vida de las personas. Algunos autores, como Lyotard, consideran que estos cambios estuvieron muy presentes en la década de los años sesenta y se consideran como el paso de la modernidad a la postmodernidad, promoviendo el paso para comenzar a hablar de la sociedad del conocimiento y de la sociedad del riesgo.

El desarrollo sostenible se considera como el paradigma idóneo de la humanidad para lograr el equilibrio en el aspecto económico, social y ambiental sin dejar de lado el componente cultural y todos los involucrados en los procesos educativos lo consideran en sus procesos operativos, como es el caso de las $\mathrm{Na}$ ciones Unidas que determina que en la educación se cifran las esperanzas para lograr un mundo más equilibrado e igualitario y lo constituye como uno de los objetivos de desarrollo del milenio, instituyendo al periodo 2005-2014 como la década de la educación para un desarrollo sostenible. El Decenio de las Naciones Unidas para la educación con miras al desarrollo sostenible pretende promover la educación como fundamento de una sociedad más viable para la humanidad e integrar el desarrollo sostenible en el sistema de enseñanza escolar a todos los niveles. El Decenio intensificará igualmente la cooperación internacional en favor 
de la elaboración y de la puesta en común de prácticas, políticas y programas innovadores de educación para el desarrollo sostenible.

Es necesario que la sociedad reciba una educación integral que permita promover una conciencia crítica a través de un enfoque interdisciplinario entre ciencia, tecnología y sociedad, donde se puedan descubrir posibilidades para minimizar el impacto ambiental de nuestras acciones y poder desarrollar habilidades para transformar el entorno haciendo uso de tradiciones, valores y hábitos, que los hombres han desarrollado a lo largo de la historia. Los adelantos tecno-científicos promueven nuevas formas de cultura. Hoy en día es muy común generar en las sociedades una conciencia forjada por el conocimiento para lograr la preservación del medio ambiente. En el contexto actual se habla de la nueva cultura del agua, una cultura que se desarrolla en torno a este recurso considerado, hasta hace poco tiempo, como renovable; esta cultura se encarga de indicar cómo utilizar y preservar este recurso, el cual es vital para la sobrevivencia de las especies.

La conservación del medio ambiente, el análisis, estudio y soluciones de todo lo que pone en peligro los ecosistemas tiene perspectivas políticas, económicas, biológicas y éticas. La educación es el camino para que las sociedades puedan construir los escenarios vitales para lograr una buena relación hombre-medio ambiente y lograr que el desarrollo tecnológico y científico permita mejorar el nivel de vida.

\section{Consideraciones finales}

En América Latina, la adopción de tecnologías occidentales ha sido una de las piedras angulares del modelo de desarrollo económico predominante (Peña, 1990). También se ha producido una expectativa en torno a la posibilidad de utilizar energías alternativas que no reproduzcan los errores cometidos por los países industrializados y que estén más de acuerdo con las necesidades y con la herencia cultural de las mayoría, así como con las exigencias de conservación del ambiente y de autodeterminación de los pueblos (Peña, 1990). Es necesario, entonces, buscar alternativas para un desarrollo tecnológico que haga posible la autodeterminación y el bienestar social. Esto, en el contexto latinoamericano, incluye, además, la preservación del medio ambiente, como lo describe Peńa (1990). En este sentido, la identificación y el estudio de problemas ecológicos específicos son necesarios para promover una conciencia ecológica que contribuya a dar forma a la política tecnológica.

El objetivo de este artículo es incentivar nuevas formas de trabajo en la relación de la tecnología con la sociedad. La premisa desde la perspectiva CTS es la inclusión de diferentes disciplinas para el análisis y de metodologías participativas 
en la resolución de problemas. Lo que pretende este enfoque es poner en cuestión la utilización de metodologías tradicionales para el control del desarrollo tecnológico y la inclusión de los aspectos sociales y ambientales a los mismos.

Por ejemplo, la evaluación constructiva de tecnologías (ECT) es una propuesta desde la perspectiva CTS. La ECT es introducida en la gestión de tecnologías, así como en su evaluación y control. Campos que se han abordado de forma tradicional sólo por disciplinas asociadas a la administración, la economía y la ingeniería. Así mismo, la gestión del riesgo plantea conceptos importantes: la medición del riesgo y la incertidumbre relacionada a los métodos de esta medición; la regulación de la incertidumbre y de la posible inequidad asociada, mediante la inclusión de la participación pública. Es decir, la inclusión de diferentes actores en la toma de decisiones - gestión del riesgo- en lugar de sólo la medición de la percepción del riesgo. En este sentido, la participación de los ciudadanos potencialmente afectados por la ciencia y la tecnología, en la gestión del riesgo, es una característica de las sociedades pluralistas.

Igualmente, se constata que la educación es un factor decisivo para lograr participar en la transformación o generación de actividades para mejor la relación hombre-ambiente. En este sentido, a corto plazo, la motivación principal desde la universidad se debe orientar hacia la educación y sensibilización en los problemas medioambientales y la formación de competencias ciudadanas para la participación pública, a diferentes niveles, en la toma de decisiones relacionadas con proyectos científico-tecnológicos. En el tema de CTS, en general -y en lo relacionado con las competencias ciudadanas para participar y avanzar hacia un desarrollo sostenible- la investigación y la pedagogía deberían salir del aula. Es decir que la investigación se debería, en cierta medida, orientar hacia la resolución de problemas locales y la inclusión de las comunidades y las organizaciones locales en el proceso.

La información que se encuentra en este trabajo constituye una herramienta para aquellas personas interesadas en la problemática ambiental y las posibles soluciones que se dan desde el punto de vista científico y tecnológico en la actualidad, con un énfasis en la interdisciplinariedad y la inclusión de diferentes actores.

Es un texto de divulgación que abre algunas preguntas. En especial la necesidad de que las sociedades se involucren en los procesos de desarrollo mediante una participación activa y racional en la toma de decisiones. En conclusión es una fuente de consulta que permitirá seguir trabajando y buscando respuestas a la forma como debe funcionar el ambiente y los medios científicos o tecnológicos para lograrlo. Dejando planteadas preguntas como: ¿cuáles son las tecnologías que deben emplearse?, ¿cuáles podrían ser los mejores modelos de desarrollo?, 
¿Cómo se mediría el impacto de las tecnologías? o ¿cómo se garantiza la participación ciudadana?

\section{Referencias}

Antal, E. (2007). "Ciencia, tecnología y sociedad en América del norte”, Revista digital universitaria, Vol. 8, (2), pp. 1 - 11, Coordinación de Publicaciones Digitales, DGSCA-UNAM, México. Disponible en Internet: http://www.revista. unam.mx/vol.8/num2/art13/int13.htm ISSN: 1607-6079.

Ahteensuu, M. (2004). "The Precautionary Principle In the Risk Management of Modern Biotechnology”, Science Studies, Vol. 17 (1), 57-65.

Avellaneda Cusaría, A. (2002). Gestión ambiental y planificación del desarrollo. El Reloj verde. Entropia-globalización-democracia-cultura, Bogotá: Ecoe Ediciones.

Beck, U. (2006). La sociedad del riesgo: hacia una nueva modernidad, Barcelona: Paidós.

Carr, S. y Ibarra, A. (2004). Las construcciones del riesgo. En: Lujan, J.L. y Echeverría, J. (editores) (2004). Gobernar los riesgos. Ciencia y valores en la sociedad del riesgo. Organización de Estados Iberoamericanos para la Educación, la Ciencia y la Cultura (OEI) / Biblioteca Nueva, Madrid, pp. $51-72$.

Cutcliffe, S.H, Goldman, S.L., Medina, M. y Sanmartin J. (editores) (1992). Estudios sobre sociedad y tecnología, Anthropos, Barcelona.

De Marchi, B. y Funtowicz, S. (2004). "La gobernabilidad del riesgo en la Unión Europea”. En: Lujan, J. L. y Echeverría, J. (editores) (2004). Gobernar los riesgos. Ciencia y valores en la sociedad del riesgo, Organización de Estados Iberoamericanos para la Educación, la Ciencia y la Cultura (OEI) / Madrid: Biblioteca Nueva, pp. 75 - 98.

Doelle M. y Sinclair J. (2006). "Time for a new approach to public participation in EA: Promoting cooperation and consensus for sustainability", Environmental Impact Assessment Review, Vol. 26, pp. 185-205

Durbin, P. (1995). "Pragmatismo y tecnología", Isegoría, No.12, pp. 80-91. Disponible en Internet: http://isegoria.revistas.csic.es/index.php/isegoria/article/ viewArticle/241. 
Funtowicz, S. y Ravetz, J. (1997). "Problemas ambientales, ciencia post normal y comunidades evaluadoras extendidas”. En: González, M.; López Cerezo, J. A. y Luján, J. L. (Editores) (1997). Ciencia, tecnología y sociedad Barcelona: Ariel, pp. $151-160$.

Genus, A. y Coles, A. M. (2005). "On Constructive Technology Assessment and Limitations on Public Participation in Technology Assessment", Technology Analysis \& Strategic Management, Vol. 17 (4), pp. 433 - 443.

Jacobson, M. y Delucchi, M. A. (2010).“Energía Sostenible: Objetivo 2030”, Investigación y Ciencia, No. 400, pp. 20-27.

Jamison, A. y Rohracher, H. (2001). "Introduction: Technology Studies and Sustainable Development”, Technology Analysis \& Strategic Management, Vol. $13(1)$, pp. $5-7$.

Lawler, D. (2003): "Las funciones técnicas de los artefactos y su encuentro con el constructivismo social en tecnología”, Revista Iberoamericana de Ciencia, Tecnologia y Sociedad - CTS, Vol. 1 (1), pp. 27-71.

Lobera, J. (2008). "Insostenibilidad: aproximación al conflicto socioecológico", Revista Iberoamericana de Ciencia, Tecnología y Sociedad CTS, Vol. 4, (11), pp. 53 - 80. Disponible en Internet: http://oeibolivia.org/files/Volumen\%204\%20\%20N\%C3\%BAmero\%2011/doss02.pdf

López Cerezo J., Méndez J., Todt, O. (1998). "Participación Pública en Política Tecnológica. Problemas y Perspectivas”, Revista Arbor, CLIX, 627, pp. 279-308.

Lujan, J. L. y Echeverría, J. (Editores) (2004). Gobernar los riesgos. Ciencia y valores en la sociedad del riesgo. Organización de Estados Iberoamericanos para la Educación, la Ciencia y la Cultura (OEI) / Biblioteca Nueva, Madrid.

Luján, J. L. y López Cerezo, J. A. (2004). “De la promoción a la regulación. El conocimiento científico en las políticas públicas de ciencia y tecnología", En: Lujan, J. L. y Echeverría, J. (editores). Gobernar los riesgos. Ciencia y valores en la sociedad del riesgo. Organización de Estados Iberoamericanos para la Educación, la Ciencia y la Cultura (OEI) / Madrid: Biblioteca Nueva. pp. 75 - 98.

Moyer, M. (2010). "La dificultades de la fusión nuclear", Investigación y Ciencia. No. 404, pp. 28-35.

Oltra Algado, C. (2006). "Sociedad y medio ambiente. Ciudadanos y científicos frente al proceso de reforma mediambiental de la sociedad", tesis doctoral, 
Universidad de Barcelona. Disponible en Internet: http://www.tesisenxarxa.net/ TDX-1005107-113944/

Palou, A. (2004). "Evaluación Científica de Riesgos y Seguridad Alimentaria en la Unión Europea”, En: Lujan, José Luis y Echeverría, Javier (editores) (2004), Gobernar los riesgos. Ciencia y Valores en la Sociedad del Riesgo. Biblioteca Nueva. Organización de los Estados Iberoamericanos. Madrid, pp. 143 - 152.

Peña Borrero, M. (1990). "Los estudios de ciencia, tecnología y sociedad en el contexto latinoamericano". En: Medina, M. y Sanmartin, J. (editores). Ciencia, Tecnología y Sociedad. Barcelona: España, pp. 125-129.

Renn, O., Webler, T. y Wiedemann, P. (editores) (1995). Fairness and Competence in Citizen Participation: Evaluating Models For Environmental Discourse, Series: Technology, Risk, and Society. An international Series in Risk Analysis, Vol. 10, Kluwer Academic Publishers.

Schot, J. (1997). "Evaluación constructiva de tecnologías: el caso de las tecnologías limpias”. En: González, M., López Cerezo, J. A. y Luján, J. L. (editores). Ciencia, tecnología y sociedad, Barcelona: Editorial Ariel, pp. 205 - 223.

Schot, J. (2001). "Towards New Forms of Participatory Technology Development”, Technology Analysis \& Strategic Management, Vol. 13, (1), pp. 39 - 52.

Shrader-Frechette, K. (1997). "Amenazas tecnológicas y soluciones democráticas”. En: González, M.; López Cerezo, J. A, y Luján, J..L.(editores). Ciencia, tecnología y sociedad. Barcelona: Ariel, pp. $225-236$.

Suárez-Herrera, M. A., Fernández Delgadillo, S. S., Garza González, M. T. y Martínez Silva, J. (2004). "Impacto de la ciencia y la tecnología en la salud y el cuidado del medio ambiente acuático", Biología, Vol. 18 (1), pp. 3-8. http:// www.dict.uh.cu/Revistas/Bio\%202004/Bi18104-1.pdf

Vilches, A. y Gil, D. (2003). Construyamos un futuro sostenible: diálogos de supervivencia, Cambridge University Press.

Vilches, A., Gil Pérez, D., Toscano, J.C. y Macías, O. (2009). “Tecnociencia para la sostenibilidad", Artículo en Internet: http://www.oei.es/decada/accion003.htm

Waks, L. (1990). "Educación en ciencia, tecnología y sociedad: orígenes, desarrollos internacionales y desafíos actuales". En: Medina M. y Sanmartín J. (editores), Ciencia, tecnologia y sociedad. Barcelona: Ariel, pp. 42-75. 
Wullschleger, S. D. y Strachi, M. (2010). "Cambio climático: un experimento controlado”, Investigación y Ciencia. No. 404, pp. 62-67.

Wynne, B. (1997). "Incertidumbre y aprendizaje ambiental: reconcebir la ciencia y la política en un paradigma preventivo”. En: González, M., López Cerezo, J. A. y Luján, J. L. (editores). Ciencia, tecnología y sociedad, Barcelona: Ariel, pp. $161-183$. 
